\title{
Sífilis congênita: prevalência e fatores associados em hospitais universitários de Pelotas
}

\section{Congenital syphilis: prevalence and related factors in university hospitals of Pelotas}

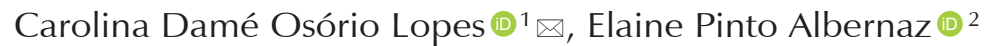 \\ ${ }^{1}$ Departamento de Pediatria da Universidade Católica de Pelotas (UCPel). Pelotas, RS, Brasil. \\ ${ }^{2}$ Departamento Materno-Infantil da Universidade Federal de Pelotas (UFPel). Pelotas, RS, Brasil.
}

RESUMO

Objetivo: Avaliar a prevalência de sífilis congênita e os fatores associados à doença em neonatos de dois hospitais universitários da cidade de Pelotas, RS.

Materiais e Métodos: Estudo transversal, retrospectivo, cujos casos de sífilis congênita foram identificados por meio das notificações compulsórias de cada hospital no período entre janeiro de 2016 a dezembro de 2017. As características dos neonatos com a doença foram comparadas àquelas dos nascidos vivos em Pelotas durante o período do estudo nestes hospitais.

Resultados: A prevalência de sífilis congênita foi de 2,4\% e a probabilidade de investigação foi maior nos filhos de gestantes mais jovens, de cor não branca e com menor escolaridade. Os neonatos investigados tiveram uma probabilidade $40 \%$ maior de nascerem com baixo peso.

Conclusão: A prevalência de sífilis congênita continua elevada e o principal impacto sobre a saúde do neonato foi o aumento da probabilidade de baixo peso ao nascer.

Palavras-chave: sífilis congênita; cuidado pré-natal; epidemiologia.

Objective: To evaluate the prevalence of congenital syphilis and the factors associated with the disease in neonates from two university hospitals in the city of Pelotas, RS.

Materials and Methods: A cross-sectional, retrospective study whose cases of congenital syphilis were identified through the compulsory notifications of each hospital from January 2016 to December 2017. The characteristics of the neonates with the disease were compared to those of the live births in Pelotas during the study period in these hospitals.

Results: The prevalence of congenital syphilis was $2.4 \%$ and the probability of investigation was higher in the offspring of younger, non-white pregnant women with lower educational level. The neonates investigated were $40 \%$ more likely to be born with low birth weight.

Conclusion: The prevalence of congenital syphilis remains high and the main impact on the newborn health was the increase in the probability of low birth weight.

Keywords: congenital syphilis; prenatal care; epidemiology. 


\section{INTRODUÇÃO}

A sífilis é uma infecção bacteriana de caráter sistêmico causada pelo Treponema pallidum (T. pallidum) e sua transmissão ocorre, predominantemente, por via sexual e vertical ${ }^{1}$. A transmissão vertical (TV) ocorre através da placenta quando a gestante portadora de sífilis não é tratada ou quando realiza o tratamento de maneira inadequada ${ }^{2}$. A infecção pelo T. pallidum, transmitida pela mãe, leva ao desenvolvimento da sífilis congênita (SC), uma doença grave que pode levar ao abortamento, parto prematuro, baixo peso ao nascer, além de acarretar outros problemas com sequelas clínicas permanentes, como alterações neurológicas e esqueléticas ${ }^{3}$.

A SC é considerada um evento sentinela em saúde, uma vez que reflete a eficácia tanto dos programas de controle quanto dos serviços que oferecem assistência prénatal. Pensando nisso, em 2007, o Ministério da Saúde (MS) lançou o Plano para Redução da Transmissão Vertical do HIV e da Sífilis no Brasil. Contudo, apesar da atenção recebida por parte dos órgãos de saúde pública nacionais e internacionais, os dados apresentados mostram que o número de casos de SC continua crescendo e permanece como um relevante problema de saúde pública ${ }^{4}$. Um estudo nacional de base hospitalar realizado no Brasil em 2012, concluiu que o controle da sífilis gestacional no país está deficiente, por outro lado, a incidência da SC, a taxa de TV e a ocorrência de desfechos negativos estão elevados ${ }^{5}$. Da mesma forma, a taxa nacional de SC aumentou 38\% nos Estados Unidos, no mesmo período, o que reforça a necessidade de um novo olhar sobre a doença ${ }^{6}$.

Dados do último Boletim Epidemiológico revelam que, em 2016, foram notificados 20.474 casos de SC no Brasil, com uma taxa de incidência de 6,8 casos/1.000 nascidos vivos, sendo as maiores taxas observadas nas regiões Sul, Sudeste e Nordeste com 7,7-7,1 e 7 casos/1.000 nascidos vivos, respectivamente, todas acima da taxa nacional. Dentre as capitais, Porto Alegre é a que apresenta a maior taxa de incidência (29,2 casos/1.000) .

Preocupados com esse cenário, a Organização Mundial da Saúde (OMS), a Organização Pan-Americana da Saúde (OPAS) e o MS lançaram como objetivo global a eliminação da SC, definida como a ocorrência de até 0,5 casos da doença para cada mil nascidos vivos ${ }^{8}$. Contudo, é notório que estamos distantes dessa meta, uma vez que a sífilis continua afetando um milhão de gestantes por ano em todo o mundo, resultando em mais de 300 mil mortes fetais e neonatais, e colocando em risco de morte prematura mais de 200 mil crianças? ${ }^{7}$.
O presente estudo tem como objetivo avaliar a prevalência de $\mathrm{SC}$ e os fatores associados à doença, em recém-nascidos (RNs) de dois hospitais universitários de referência na cidade de Pelotas-RS, que juntos, são responsáveis pela realização da maioria dos partos do município. Esta avaliação se justifica pelo número crescente de casos de sífilis no país, entre elas, a congênita.

\section{MATERIAIS E MÉTODOS}

Estudo de delineamento transversal, retrospectivo, realizado em dois hospitais universitários da cidade de Pelotas-RS, que integram a rede do Sistema Único de Saúde (SUS), além de ser referência para as gestações de alto risco. Pelotas é a terceira cidade mais populosa do estado, com 328.275 habitantes (Censo 2010), conta com 51 Unidades Básicas de Saúde, 55 equipes de estratégia de saúde da família e mais três hospitais que prestam assistência à gestante, além dos dois hospitais universitários incluídos no estudo.

A população do estudo foi composta por recém-nascidos com SC nascidos no Hospital Universitário São Francisco de Paula (HUSFP) e no Hospital Escola da Universidade Federal de Pelotas (HE- UFPEL) no período de janeiro de 2016 a dezembro de 2017. Foram excluídos do estudo os casos de aborto e natimortos.

O cálculo do tamanho da amostra foi realizado utilizando a prevalência estimada de SC de $2 \%$, nível de confiança de $95 \%$ e margem de erro de 1,5 pontos percentuais. Os casos de sífilis materna e SC foram identificados por meio das notificações compulsórias entregues à Comissão de Controle de Infecção Hospitalar (CCIH) de cada hospital neste período.

As variáveis de exposição coletadas foram: a) Características sociodemográficas maternas: idade (anos completos), cor da pele (branca, preta, amarela, parda, indígena), escolaridade (sem estudo, Fundamental I, Fundamental II, Médio, Superior incompleto, Superior completo) e ocupação; b) Assistência pré-natal: número de consultas, momento em que foi realizado o diagnóstico de sífilis ( 10 trimestre, 2 o trimestre, 3o trimestre, parto), tratamento recebido pela gestante e pelo parceiro (adequado, inadequado, não realizado, ignorado) e coinfecção pelo vírus HIV (sim ou não); c) Características do $\mathrm{RN}$ : sexo, peso ao nascer (em gramas) e idade gestacional ao nascimento (ultrassonografia precoce ou método de Capurro).

Foi considerado tratamento materno adequado aquele realizado com Penicilina, na dose adequada à fase da doença, finalizado pelo menos 30 dias antes do parto, tendo sido o parceiro concomitantemente tratado, de acordo com as normas do MS para prevenção da transmissão vertical de sífilis². 
As variáveis de desfecho estudadas foram: presença de sintomas de SC ao nascimento, titulação do VDRL, radiografia de ossos longos (normal, alterada, não realizada), líquor (normal, alterado, não realizado), hemograma (normal, alterado, não realizado), investigação adequada do RN (sim, não, sem dados para avaliação), tratamento do RN (sim ou não), medicamento usado (Penicilina Benzatina, Penicilina Cristalina, Penicilina Procaína, outra classe de antibiótico) e tempo de hospitalização (dias de internação).

Foram considerados sintomáticos os neonatos que apresentaram hepatoesplenomegalia, lesões cutâneas, rinite serosanguinolenta, sofrimento respiratório com ou sem pneumonia, icterícia, pseudoparalisia e edema². Quanto aos exames, foram considerados alterados: radiografia com sinais de periostite, osteíte e osteocondrite; líquor com VDRL positivo, proteínas acima de 150 ou leucócitos acima de 25 e hemograma com anemia, plaquetopenia, leucocitose ou leucopenia ${ }^{3}$. A investigação do $\mathrm{RN}$ foi realizada levando em consideração a adequação do tratamento materno e seguindo o fluxograma estabelecido pelo $\mathrm{MS}^{2}$.

Os dados foram analisados com o programa SPSS 21.0. Inicialmente foram descritas as características da amostra e, a seguir, foram feitas análises bivariadas utilizando o teste do Qui-quadrado, para obtenção das razões de prevalência (RP). As características dos RNs com SC que compuseram a amostra foram comparadas com aquelas dos nascidos vivos em Pelotas durante o período do estudo, nestes hospitais. Os dados destes foram obtidos junto ao Sistema de Informação sobre Nascidos Vivos (SINASC). Foram consideradas estatisticamente significativas as associações com valor de $p$ inferior a 0,05 .

Este estudo foi aprovado pelo Comitê de Ética em Pesquisa das duas instituições participantes, pareceres número 2.969 .452 e 2.792.188. Foi utilizada uma ficha espelho para coleta de informações que foram extraídas dos prontuários das mães e dos bebês com diagnóstico de sífilis, conforme termo de compromisso para utilização de dados. $\mathrm{O}$ sigilo e o anonimato dos participantes foram assegurados durante a pesquisa.

\section{RESULTADOS}

No período de janeiro de 2016 a dezembro de 2017, ocorreram 10.667 nascimentos na cidade de Pelotas, sendo 5.641 (53\%) nos dois hospitais incluídos no estudo. Foram identificadas 193 gestantes com diagnóstico de sífilis gestacional. Entre os recém-nascidos, 55 tiveram VDRL negativo, 138 apresentaram VDRL positivo e necessitaram de avaliação adicional, o que levou a 2,4\% de casos suspeitos de sífilis congênita. Houve acesso a todos os prontuários e foram identificados nas tabelas os percentuais de ausência de informação para algumas das variáveis estudadas.

Na Tabela 1, encontram-se as características das gestantes que tiveram filhos no período, conforme os dados do SINASC. Já a Tabela 2, apresenta a distribuição das gestantes com sífilis gestacional de acordo com as características sociodemográficas e variáveis relacionadas ao pré-natal, dados estes coletados por meio dos prontuários. A maioria das gestantes estava na faixa etária entre 21 e 30 anos $(50,8 \%)$, era de cor branca $(57,5 \%)$ e completou o ensino fundamental $(65,3 \%)$.

Tabela 1. Características dos nascimentos no município e nos hospitais do estudo.

\begin{tabular}{|c|c|c|}
\hline Variáveis & $\begin{array}{c}\text { Todos hospitais } \\
\text { n (\%) }\end{array}$ & $\begin{array}{c}\text { Hospitais universitários } \\
\text { n (\%) }\end{array}$ \\
\hline \multicolumn{3}{|l|}{ Idade materna } \\
\hline Até 20 anos & $1878(17,6)$ & $943(16,7)$ \\
\hline 21-30 anos & $4970(46,6)$ & $2498(44,3)$ \\
\hline Acima de 30 anos & $3819(35,8)$ & $2200(39,0)$ \\
\hline \multicolumn{3}{|l|}{ Cor materna } \\
\hline Branca & $8224(77,1)$ & $4190(74,3)$ \\
\hline Não Branca & $1967(18,4)$ & $1119(19,8)$ \\
\hline Ignorada & $476(4,5)$ & $332(5,9)$ \\
\hline \multicolumn{3}{|l|}{ Escolaridade } \\
\hline Sem escolaridade & $10(0,1)$ & $7(0,1)$ \\
\hline Fundamental I & $351(3,3)$ & $231(4,1)$ \\
\hline Fundamental II & $3200(30,0)$ & $1615(28,6)$ \\
\hline Ensino Médio & $3956(37,1)$ & 1974 (35) \\
\hline Ensino Superior & $2924(27,4)$ & $1656(29,4)$ \\
\hline Ignorado & $226(2,1)$ & $158(2,8)$ \\
\hline \multicolumn{3}{|l|}{ Ocupação } \\
\hline Do lar & $4711(44,2)$ & $2570(45,6)$ \\
\hline Estudante & $699(6,6)$ & $313(5,5)$ \\
\hline Trabalha fora de casa & $4946(46,4)$ & $2561(45,4)$ \\
\hline Ignorado & $311(2,9)$ & $197(3,5)$ \\
\hline \multicolumn{3}{|l|}{ Número de consultas } \\
\hline 0 a 5 consultas & $1372(12,9)$ & $808(14,3)$ \\
\hline 6 ou mais consultas & $7305(68,5)$ & $2974(52,7)$ \\
\hline Ignorado & $1990(18,7)$ & $1859(33,0)$ \\
\hline \multicolumn{3}{|l|}{ Peso ao nascer } \\
\hline$<2500 \mathrm{~g}$ & $1231(11,5)$ & $1074(19,0)$ \\
\hline$\geq 2500 \mathrm{~g}$ & $9436(88,5)$ & $4567(81,0)$ \\
\hline Total & $10667(100)$ & $5641(100)$ \\
\hline
\end{tabular}

Considerando o número de consultas, 52,8\% realizaram mais de seis consultas de pré-natal, sendo o VDRL o exame mais empregado no diagnóstico materno (96,4\%). Aproximadamente 55\% dos diagnósticos ocorreram no terceiro trimestre ou no momento do parto. Quanto ao tratamento, apenas 84 gestantes $(43,5 \%)$ e 62 parceiros $(32,1 \%)$ receberam o tratamento adequado. Entre as gestantes diagnosticadas com sífilis, 7,3\% apresentaram coinfecção pelo vírus HIV. 
Tabela 2. Distribuição das gestantes com sífilis conforme características sociodemográficas e variáveis relacionadas ao pré-natal.

\begin{tabular}{|c|c|}
\hline Variáveis & n (\%) \\
\hline \multicolumn{2}{|l|}{ Idade materna } \\
\hline Até 20 anos & $64(33,2)$ \\
\hline 21 a 30 anos & $98(50,8)$ \\
\hline Acima de 30 anos & $31(16,1)$ \\
\hline \multicolumn{2}{|l|}{ Cor materna } \\
\hline Branca & $111(57,5)$ \\
\hline Não branca & $82(42,5)$ \\
\hline \multicolumn{2}{|l|}{ Escolaridade } \\
\hline Fundamental I & $17(8,8)$ \\
\hline Fundamental II & $109(56,5)$ \\
\hline Ensino Médio & $57(29,5)$ \\
\hline Ensino Superior & $5(2,6)$ \\
\hline Ignorado & $5(2,6)$ \\
\hline \multicolumn{2}{|l|}{ Ocupação } \\
\hline Do lar & $117(60,6)$ \\
\hline Estudante & $21(10,9)$ \\
\hline Trabalha fora de casa & $54(28,0)$ \\
\hline Ignorado & $1(0,5)$ \\
\hline \multicolumn{2}{|c|}{ Número de consultas pré-natal } \\
\hline 0 a 5 consultas & $77(39,9)$ \\
\hline 6 ou mais consultas & $102(52,8)$ \\
\hline Ignorado & $14(7,3)$ \\
\hline \multicolumn{2}{|l|}{ Momento do diagnóstico } \\
\hline $1^{\circ}$ trimestre & $48(24,9)$ \\
\hline $2^{\circ}$ trimestre & $36(18,7)$ \\
\hline $3^{\circ}$ trimestre & $49(25,4)$ \\
\hline Parto & $57(29,5)$ \\
\hline Ignorado & $3(1,6)$ \\
\hline \multicolumn{2}{|l|}{ Tratamento da gestante } \\
\hline Adequado & $84(43,5)$ \\
\hline Inadequado & $50(25,9)$ \\
\hline Não realizado & $56(29,0)$ \\
\hline Ignorado & $3(1,6)$ \\
\hline \multicolumn{2}{|l|}{ Tratamento do parceiro } \\
\hline Adequado & $62(32,1)$ \\
\hline Inadequado & $9(4,7)$ \\
\hline Não realizado & $83(43,0)$ \\
\hline Ignorado & $39(20,2)$ \\
\hline Total & $193(100)$ \\
\hline
\end{tabular}

Na Tabela 3, são apresentadas as características dos recém-nascidos com suspeita de sífilis congênita. Dos 193 neonatos avaliados, $26,4 \%$ apresentaram peso ao nascer inferior a $2500 \mathrm{~g}$ e $27,5 \%$ nasceram antes de 37 semanas de idade gestacional. A grande maioria era assintomática ao nascimento $(94,3 \%)$ e $71,5 \%$ tinham VDRL reagente no sangue.

Entre os bebês analisados, 10,4\% apresentaram alteração radiográfica e 12,4\% alteração liquórica, sendo a investigação adequada em 90,7\% dos casos. Com relação ao tratamento,
45,1\% dos RNs receberam Penicilina Cristalina por 10 dias e $35,2 \%$ Penicilina Benzatina em dose única. O tempo de internação foi superior a sete dias em $64,8 \%$ dos casos. Ao avaliar o impacto da adequação do tratamento materno nos desfechos apresentados nesta tabela, só houve associação estatisticamente significativa com o VDRL e o hemograma do RN. Os filhos de mães que não receberam tratamento ou foram inadequadamente tratadas, apresentaram uma probabilidade duas vezes maior de positividade do VDRL $(\mathrm{RP}=2,27 ; p=0,001)$ e maior probabilidade de alteração do hemograma ( $R P=1,13 ; p=0,003)$; (dados não apresentados na tabela).

Tabela 3. Distribuição dos recém-nascidos com suspeita de sífilis congênita de acordo com as características do nascimento, investigação e tratamento.

\begin{tabular}{|c|c|}
\hline Variáveis & n (\%) \\
\hline \multicolumn{2}{|l|}{ Sexo } \\
\hline Masculino & $91(47,2)$ \\
\hline Feminino & $102(52,8)$ \\
\hline \multicolumn{2}{|l|}{ Peso ao nascer } \\
\hline$<2500 \mathrm{~g}$ & $51(26,4)$ \\
\hline$\geq 2500 \mathrm{~g}$ & $142(73,6)$ \\
\hline \multicolumn{2}{|l|}{ Idade gestacional } \\
\hline Até 36 semanas & $53(27,5)$ \\
\hline$\geq 37$ semanas & $140(72,5)$ \\
\hline \multicolumn{2}{|l|}{ Presença de sintomas } \\
\hline Sim & $11(5,7)$ \\
\hline Não & $182(94,3)$ \\
\hline \multicolumn{2}{|l|}{ VDRL sérico } \\
\hline Não reagente & $55(28,5)$ \\
\hline Reagente & $138(71,5)$ \\
\hline \multicolumn{2}{|l|}{ Radiografia de ossos longos } \\
\hline Normal & $138(71,5)$ \\
\hline Alterado & $20(10,4)$ \\
\hline Não realizado & $35(18,1)$ \\
\hline \multicolumn{2}{|l|}{ Hemograma } \\
\hline Normal & $136(70,5)$ \\
\hline Alterado & $23(11,9)$ \\
\hline Não realizado & $34(17,6)$ \\
\hline \multicolumn{2}{|l|}{ Líquor } \\
\hline Normal & $121(62,7)$ \\
\hline Alterado & $24(12,4)$ \\
\hline Não realizado & $48(24,9)$ \\
\hline \multicolumn{2}{|l|}{ Investigação } \\
\hline Adequada & $175(90,7)$ \\
\hline Não adequada & $18(9,3)$ \\
\hline \multicolumn{2}{|l|}{ Tratamento } \\
\hline Penicilina cristalina & $87(45,1)$ \\
\hline Penicilina procaína & $8(4,1)$ \\
\hline Penicilina benzatina & $68(35,2)$ \\
\hline Outra classe de antibiótico & $23(11,9)$ \\
\hline Não realizado & $7(3,6)$ \\
\hline Total & $193(100)$ \\
\hline
\end{tabular}


Tabela 4. Fatores sociodemográficos relacionados à investigação de sífilis congênita entre os nascidos nos hospitais universitários.

\begin{tabular}{|c|c|c|c|c|}
\hline Variáveis & $\mathbf{n}$ & $\begin{array}{l}\text { Investigação de sífilis } \\
\text { congênita (\%) }\end{array}$ & RP (IC95\%) & Valor de $p^{*}$ \\
\hline \multicolumn{5}{|l|}{ Idade materna } \\
\hline Até 20 anos & 943 & 6,3 & $5,25(3,36-8,34)$ & 0,001 \\
\hline 21-30 anos & 2498 & 3,6 & $3,05(1,98-4,70)$ & \\
\hline Acima de 30 anos & 2200 & 1,2 & 1,00 & \\
\hline \multicolumn{5}{|l|}{ Cor materna** } \\
\hline Não Branca & 1119 & 6,1 & $2,68(1,98-3,63)$ & 0,001 \\
\hline Branca & 4190 & 2,3 & 1,00 & \\
\hline \multicolumn{5}{|l|}{ Escolaridade materna*** } \\
\hline Até fundamental I & 238 & 5,0 & $2,51(1,38-4,55)$ & 0,001 \\
\hline Fundamental II & 1615 & 5,0 & $2,49(1,83-3,40)$ & \\
\hline Ensino Médio ou Superior & 3630 & 2,0 & 1,0 & \\
\hline Total & 5641 & 3,1 & & \\
\hline
\end{tabular}

O SINASC não permite a identificação das gestantes, portanto, não foi possível uma análise adequada dos fatores associados à ocorrência de SC. Buscou-se localizar no banco de dados do SINASC os neonatos que foram investigados para SC por meio das variáveis data e peso de nascimento, idade, cor e escolaridade materna e número de consultas de pré-natal. Desta forma, foram identificados 175 dos 193 neonatos investigados. Estes 175 neonatos foram comparados aos demais nascidos nos hospitais universitários. Verificou-se que a probabilidade de investigação para $\mathrm{SC}$ foi maior nos filhos de gestantes mais jovens $(\mathrm{RP}=5,25)$, de cor não branca $(R P=2,68)$ e com menor escolaridade $(R P=2,51)$. Estas diferenças foram estatisticamente significativas (Tabela 4).

Os RNs investigados para SC tiveram uma probabilidade $40 \%$ maior de nascerem com baixo peso ( $R P=1,40$, $p=0,013)$. Tendo em vista que a informação sobre o número de consultas pré-natais constava como ignorada para 33\% da amostra referente aos hospitais universitários no SINASC, esta não foi analisada.

\section{DISCUSSÃO}

A prevalência de sífilis gestacional entre as mulheres que tiveram filhos nascidos vivos foi 3,4 e 2,4\% dos recém-nascidos tinham suspeita de SC, valores acima dos encontrados por Domingues et al. ${ }^{9}$. Esses resultados também são superiores aos descritos no último boletim epidemiológico de 20177, o que demonstra que, apesar da sífilis ser uma doença com diagnóstico e tratamento bem estabelecidos e de baixo custo, sua erradicação continua sendo um desafio para os gestores e profissionais de saúde.
Outro aspecto relevante diz respeito à coinfecção, já que a sífilis pode facilitar a TV do HIV, fato especialmente preocupante em países como o Brasil em que as duas doenças são comuns. Neste estudo, 7,3\% das gestantes com sífilis apresentaram coinfecção pelo vírus HIV, valores inferiores aos encontrados na literatura ${ }^{10,11}$.

O perfil das gestantes com sífilis no presente estudo é semelhante aos descritos na literatura nacional e internacional, sendo mais frequente na faixa etária mais jovem, com menor escolaridade e que não tem trabalho remunerado ${ }^{12-15}$. Embora não seja uma doença restrita às classes menos favorecidas, estes marcadores de baixo nível socioeconômico podem sinalizar que esta camada da população tem menos acesso aos serviços de saúde e, consequentemente, uma assistência pré-natal inadequada, contribuindo para o aumento da TV nesse grupo ${ }^{16}$.

O MS preconiza que o início da assistência pré-natal deve ocorrer no primeiro trimestre e contar com o número mínimo de seis consultas ${ }^{17}$. Observamos que mais da metade das gestantes apresentaram número de consultas adequado, apesar disso, a maioria dos diagnósticos foram realizados de forma tardia, no terceiro trimestre de gestação ou no momento do parto, o que corrobora os achados de outros autores, indicando que assegurar o número mínimo de consultas não é o suficiente para garantir a qualidade da assistência ${ }^{5,13,14,18,19}$.

Assim como encontrado por Magalhães et al. ${ }^{13}$, menos da metade das gestantes com sífilis realizou tratamento adequado, fato preocupante, pois demostra que, apesar das políticas públicas instituídas até o momento, não houve mudança significativa neste cenário. Estas falhas na assistência 
impactam diretamente na saúde da gestante e do concepto, resultando na perpetuação de altas taxas de TV e mantendo a prevalência de $\mathrm{SC}$ bem acima dos 0,5 casos/1.000 nascidos vivos estipulados como objetivo global.

Quanto aos recém-nascidos com suspeita de SC, 26,4\% nasceram com baixo peso e $27,5 \%$ foram prematuros, valores bem acima dos encontrados por Barros et al. ${ }^{20}$ na coorte de nascimentos da cidade de Pelotas, onde a frequência de prematuridade foi de $15 \%$ e a de baixo peso ao nascer foi de $10 \%$, acima também dos percentuais de baixo peso no período do estudo, conforme o SINASC (11,5\%). Apesar dos RNs expostos à infecção materna estarem sob maior risco de prematuridade e baixo peso, mais de $90 \%$ dos casos foram assintomáticos ao nascimento, achado semelhante ao descrito por Lafetá et al. ${ }^{18}$ e que demonstra que o diagnóstico de SC é um processo complexo e requer alta suspeição clínica.

Dos RNs investigados, 10,4\% apresentaram alterações radiológicas, principalmente rarefações em membros inferiores, valores acima dos descritos na literatura ${ }^{21,22}$. Por outro lado, o líquor apresentou alteração em 12,4\% dos casos e em aproximadamente $25 \%$ a coleta não foi realizada, índices inferiores aos encontrados por Cardoso et al..$^{22}$ no estado do Ceará.

Este estudo buscou não só avaliar a ocorrência de SC, como também a adequação no processo investigação e terapêutica. Diferentes autores identificaram falhas no processo de investigação, entretanto, neste estudo, a investigação foi considerada inadequada em apenas 9,3\% dos neonatos, sendo a falha na coleta de líquor a causa mais frequente de inadequação ${ }^{22,23}$. Este achado pode ser atribuído ao fato de que as duas instituições envolvidas são universitárias e seguem literalmente o protocolo de investigação proposto pelo MS, o que sinaliza um avanço positivo no cuidado com o RN e no diagnóstico da SC e reforça a importância da utilização dos protocolos de investigação e tratamento do MS.

Em relação ao tratamento do $\mathrm{RN}$, o MS preconiza que seja realizado com Penicilina Cristalina, Procaína ou Benzatina, de acordo com avaliação clínica e laboratorial realizada e, afirma ainda, que não há evidências que comprovem a eficácia da Ceftriaxona no tratamento da $\mathrm{SC}^{3}$. Constatou-se que $11,9 \%$ dos RNs investigados, receberam Ceftriaxona como droga alternativa para o tratamento da infecção congênita, condição que pode ser justificada devido às dificuldades enfrentadas pelo desabastecimento mundial de Penicilina em 2016, os outros $84,4 \%$ dos casos foram tratados com Penicilina ${ }^{24}$.

Lacunas no processo de diagnóstico e tratamento da gestante, além das consequências já descritas à saúde do neonato, causam também impacto financeiro ao sistema e acabam por onerar o SUS, uma vez que prolongam o tempo de internação dos bebês. Dos 193 RNs avaliados neste estudo, $64,8 \%$ permaneceram internados por mais de sete dias, frequência inferior às encontradas por pesquisadores americanos e brasileiros, mas ainda assim, três vezes superior ao tempo de internação esperado para um $\mathrm{RN}$ a termo e sem intercorrências6,22.

Entre as limitações do estudo, ressalta-se que o cálculo da prevalência de SC foi de casos suspeitos, ou seja, bebês que foram expostos à doença e apresentaram VDRL positivo ao nascimento e, portanto, necessitaram de investigação adicional. Não foi realizada pesquisa do T. pallidum, e, por se tratar de um estudo transversal, não houve seguimento dos RNs até os 18 meses para confirmação do diagnóstico, desta forma, a prevalência da doença pode ter sido superestimada. Por outro lado, os casos mais graves de infecção congênita que resultaram em abortamento e óbito fetal não foram incluídos na amostra por não serem objetivo desta pesquisa. Além disso, devido à utilização de dados secundários, para algumas variáveis houve ausência de informação, o que reforça a importância do preenchimento adequado dos prontuários e documentos de notificação.

Conclui-se que a prevalência de SC nos hospitais universitários de Pelotas está acima da média observada na região Sul do Brasil. Os principais fatores associados à doença foram idade, cor e escolaridade materna e o maior impacto gerado sobre a saúde do neonato foi o aumento dos índices de baixo peso ao nascer. Por outro lado, houve um avanço positivo no cuidado com o $\mathrm{RN}$, demonstrado pelo baixo índice de falhas no processo de investigação e pelas altas taxas de adequação ao tratamento. Manter a vigilância em relação à ocorrência da sífilis é fundamental e novos estudos deveriam incluir a adequação da investigação e do tratamento, visando a prevenção de sequelas aos neonatos. Novas estratégias devem ser pensadas no intuito de investir em campanhas de conscientização e captação precoce das gestantes, visando reduzir a TV e, consequentemente, a prevalência de SC.

\section{REFERÊNCIAS}

1. Brasil. Ministério da Saúde. Secretaria de Vigilância em Saúde. Departamento de Vigilância, Prevenção e Controle das Doenças Sexualmente Transmissíveis, Aids e Hepatites Virais. Manual técnico para diagnóstico da sífilis. Brasília: Ministério da Saúde; 2016. https://doi.org/10.26512/2016.02.d.20359

2. Brasil. Ministério da Saúde. Secretaria de Vigilância em Saúde. Departamento de DST, Aids e Hepatites Virais. Protocolo Clínico e Diretrizes Terapêuticas (PCDT): atenção integral às pessoas com Infecções Sexualmente Transmissíveis (IST). Brasília: Ministério da Saúde; 2015. https://doi.org/10.26512/2016.02.d.20359 
3. Brasil. Ministério da Saúde. Comissão Nacional de Incorporação de Tecnologias no SUS. (CONITEC). Protocolo clínico e diretrizes terapêuticas para prevenção da transmissão vertical de HIV, sífilis e hepatites virais. Brasília: Ministério da Saúde; 2017. https://doi. org/10.11606/t.5.2019.tde-17042019-092843

4. Brasil. Ministério da Saúde. Secretaria de Atenção à Saúde. Departamento de Ações Programáticas e Estratégicas. Atenção à saúde do recém-nascido: guia para os profissionais de saúde: cuidados gerais. Brasília: Ministério da Saúde; 2011. https://doi. org/10.18554/reas.v5i2.1426

5. Domingues RM, Leal MC. Incidence of congenital syphilis and factors associated with vertical transmission: data from the Birth in Brazil study. Cad Saúde Pública. 2016;32(6):e00082415. http:// dx.doi.org/10.1590/0102-311X00082415

6. Bowen V, Su J, Torrone E, Kidd S, Weinstock H. Increase in incidence of congenital syphilis - United States, 2012-2014. MMWR Morb Mortal Wkly Rep. 2015;64(44):1241-5. https://doi. org/10.15585/mmwr.mm6444a3

7. Brasil. Ministério da Saúde. Secretaria de Vigilância em Saúde. Boletim epidemiológico: sífilis 2017;48(36):1-42. https://doi. org/10.7476/9788575416068.0002

8. Brasil. Ministério da Saúde. Transmissão vertical do HIV e sífilis: estratégias para redução e eliminação. Brasília: Ministério da Saúde; 2014. https://doi.org/10.1590/s003489102011000400026

9. Domingues RMSM, Saracen V, Hartz ZMA, Leal MC. Sífilis congênita: evento sentinela da qualidade da assistência pré-natal. Rev Saúde Pública. 2013;47(1):147-57. http://dx.doi.org/10.1590/ S0034-89102013000100019

10. Yeganeh N, Watts HD, Camarca M, Soares G, Joao E, Pilotto JH, Gray G, Theron G, Santos B, Fonseca R, Kreitchmann R, Pinto J, Mussi-Pinhata M, Ceriotto M, Machado DM, Grinzstein B, Veloso VG, Morgado MG, Bryson Y, Mofenson LM, Nielsen-Saines K; NICHD HPTN 040P1043 Study Team. Syphilis in HIV-infected mothers and infants: results from the NICHD/HPTN 040 study. Pediatr Infect Dis J. 2015;34(3):e52-7. http://dx.doi.org/10.1097/ INF.0000000000000578

11. Acosta LMW, Gonçalves TR, Barcellos NT. Coinfecção HIV/ sífilis na gestação e transmissão vertical do HIV: um estudo a partir de dados da vigilância epidemiológica. Rev Panam Salud Publica. 2016;40(6):435-42. https://doi.org/10.1590/s102049892006000300005

12. Soares LG, Zarpellon B, Soares LG, Baratieri T, Lentsck MH, Mazza VA. Gestational and congenital syphilis: maternal, neonatal characteristics and outcome of cases. Rev Bras Saúde Mater Infant. 2017;17(4):781-9. http://dx.doi.org/10.1590/180693042017000400010

13. Magalhães DMS, Kawaguchi IAL, Dias A, Calderon IMP. Sífilis materna e congênita: ainda um desafio. Cad Saúde Pública. 2013;29(6):1109-20. http://dx.doi.org/10.1590/S0102$311 \times 2013000600008$
14. Campos ALA, Araújo MAL, Melo SP, Gonçalves MLC. Epidemiologia da sífilis gestacional em Fortaleza, Ceará, Brasil: um agravo sem controle. Cad Saúde Pública. 2010;26(9):1747-55. http://dx.doi.org/10.1590/S0102-311X2010000900008

15. Dou L, Wang X, Wang F, Wang Q, Qiao Y, Su M, Jin X, Qiu J, Song L, Wang A. Epidemic profile of maternal syphilis in China in 2013. BioMed Res Int. 2016;2016:9194805. http://dx.doi. org/10.1155/2016/9194805

16. Araújo CL, Shimizu HE, Sousa AIA, Hamann EM. Incidência da sífilis congênita no Brasil e sua relação com a Estratégia Saúde da Família. Rev Saúde Pública. 2012;46(3):479-86. http://dx.doi. org/10.1590/S0034-89102012000300010

17. Brasil. Ministério da Saúde. Portaria n 570, de $1^{\circ}$ junho 2000 [Internet]. Brasília: Ministério da Saúde; 2000 [capturado em 15 out. 2019]. Disponível em: http://bvsms.saude.gov.br/bvs/ saudelegis/gm/2000/prt0570_01_06_2000_rep.html. https://doi. org/10.5327/z1679443520190289

18. Lafetá KR, Martelli Júnior H, Silveira MF, Paranaíba LM. Maternal and congenital syphilis underreported and difficult to control. Rev Bras Epidemiol. 2016;19(1):63-74. http://dx.doi. org/10.1590/1980-5497201600010006

19. Lima BGC, Costa MCN, Dourado MIC. Avaliação da qualidade do rastreamento de HIV/aids e sífilis na assistência pré-natal. Epidemiol Serv Saúde. 2008;17(2):125-7.

20. Barros AJ, Santos IS, Victora CG, Albernaz EP, Domingues MR, Timm IK, Matijasevich A, Bertoldi AD, Barros FC. Coorte de nascimentos de Pelotas, 2004: metodologia e descrição. Rev Saúde Pública. 2006;40(3):402-13. http://dx.doi.org/10.1590/ S0034-89102006000300007

21. Muricy CL, Pinto Júnior VL. Congenital and maternal syphilis in the capital of Brazil. Rev Soc Bras Med Trop. 2015;48(2):216-9. http://dx.doi.org/10.1590/0037-8682-0168-2014

22. Cardoso ARP, Araújo MAL, Cavalcante MS, Frota MA, Melo SP. Análise dos casos de sífilis gestacional e congênita nos anos de 2008 a 2010 em Fortaleza, Ceará, Brasil. Ciênc Saúde Coletiva. 2018;23(2):563-74. http://dx.doi.org/10.1590/141381232018232.01772016

23. Patel SJ, Klinger EJ, O'Toole D, Schillinger JA. Missed opportunities for preventing congenital syphilis infection in New York City. Obstet Gynecol. 2012;120(4):882-8. http://dx.doi.org/10.1097/ AOG.0b013e31826ac25f

24. Taylor MM, Nurse-Findlay S, Zhang X, Hedman L, Kamb ML, Broutet $M$, Kiarie J. Estimating benzathine penicillin need for the treatment of pregnant women diagnosed with syphilis during antenatal care in high-morbidity countries. PLoS One. 2016;11(7):e0159483. http://dx.doi.org/10.1371/journal. pone. 0159483 


\section{Autoras:}

CAROLINA DAMÉ OSÓRIO LOPES

aMédica. Mestre em Saúde da Mulher, Criança e Adolescente pela Universidade

Católica de Pelotas (UCPel). Professora Adjunta do Departamento de Pediatria da

uCPel.

Orcid: http://orcid.org/0000-0003-2009-6188

E-mail: caodame@hotmail.com

ELAINE PINTO ALBERNAZ

bMédica. Doutora em Epidemiologia e Professora Titular do Programa de Pós-

Graduação em Saúde da Mulher, Criança e Adolescente da UCPel. Professora Adjunta

do Departamento Materno-Infantil da Universidade Federal de Pelotas (UFPel).

Orcid: http://orcid.org/0000-0002-5016-9651

E-mail: elaine.albernaz@pq.cnpq.br 